University of Wollongong

Research Online

Faculty of Science, Medicine and Health -

Papers: Part B

Faculty of Science, Medicine and Health

2020

The relevance of whole grain food definitions in estimation of whole grain intake: a secondary analysis of the National Nutrition and Physical Activity Survey 2011-2012

\author{
Katrina Kissock \\ University of Wollongong, krk981@uowmail.edu.au \\ Elizabeth Neale \\ University of Wollongong, elizan@uow.edu.au \\ Eleanor J. Beck \\ University of Wollongong, eleanor@uow.edu.au
}

Follow this and additional works at: https://ro.uow.edu.au/smhpapers1

Publication Details Citation

Kissock, K., Neale, E., \& Beck, E. J. (2020). The relevance of whole grain food definitions in estimation of whole grain intake: a secondary analysis of the National Nutrition and Physical Activity Survey 2011-2012. Faculty of Science, Medicine and Health - Papers: Part B. Retrieved from https://ro.uow.edu.au/ smhpapers1/1378

Research Online is the open access institutional repository for the University of Wollongong. For further information contact the UOW Library: research-pubs@uow.edu.au 


\title{
The relevance of whole grain food definitions in estimation of whole grain intake: a secondary analysis of the National Nutrition and Physical Activity Survey 2011-2012
}

\author{
Abstract \\ Objective:
}

To determine the impacts of using a whole grain food definition on measurement of whole grain intake compared with calculation of total grams of intake irrespective of the source. Design:

The Australian whole grain database was expanded to identify foods that comply with the Healthgrain whole grain food definition ( $\geq 30 \%$ whole grains on a dry weight basis, whole grain ingredients exceeds refined grain and meeting accepted standards for healthy foods based on local regulations). Secondary analysis of the National Nutrition and Physical Activity Survey (NNPAS) 2011-2012 dietary intake data included calculation of whole grain intakes based on intake from foods complying with the Healthgrain definition. These were compared with intake values where grams of whole grain in any food had been included. Setting:

Australia. Participants:

Australians ( $\geq 2$ years) who participated in the NNPAS 2011-2012 (n 12 153). Results:

Following expansion of the whole grain database, 214 of the 609 foods containing any amount of whole grain were compliant with the Healthgrain definition. Significant mean differences (all $P<0.05$ ) of $2 \cdot 84-6 \cdot 25 \mathrm{~g} / \mathrm{d}$ of whole grain intake (5.91-9.44 g/d energy adjusted) were found when applying the Healthgrain definition in comparison with values from foods containing any whole grain across all age groups. Conclusions:

Application of a whole grain food definition has substantial impact on calculations of population whole grain intakes. While use of such definitions may prove beneficial in settings such as whole grain promotion, the underestimation of total intake may impact on identification of any associations between whole grain intake and health outcomes.

\section{Keywords}

definitions, food, nutrition, national, analysis, secondary, grain, whole, relevance, 2011-2012, survey, activity, physical, intake:, estimation

\section{Publication Details}

Kissock, K. R., Neale, E. P. \& Beck, E. J. (2020). The relevance of whole grain food definitions in estimation of whole grain intake: a secondary analysis of the National Nutrition and Physical Activity Survey 2011-2012. Public Health Nutrition, 23 (8), 1307-1319. 


\title{
The relevance of whole grain food definitions in estimation of whole grain intake: a secondary analysis of the National Nutrition and Physical Activity Survey 2011-2012
}

\author{
Katrina R Kissock ${ }^{1,2, *}$, Elizabeth P Neale $\mathrm{e}^{1,2}$ and Eleanor J Beck ${ }^{1,2}$ \\ 'School of Medicine, Faculty of Science, Medicine and Health, University of Wollongong, Wollongong, \\ NSW, Australia: ${ }^{2}$ llawarra Health and Medical Research Institute, University of Wollongong, Wollongong, \\ NSW, Australia
}

Submitted 12 May 2019: Final revision received 13 0ctober 2019: Accepted 18 0ctober 2019

\begin{abstract}
Objective: To determine the impacts of using a whole grain food definition on measurement of whole grain intake compared with calculation of total grams of intake irrespective of the source.

Design: The Australian whole grain database was expanded to identify foods that comply with the Healthgrain whole grain food definition $(\geq 30 \%$ whole grains on a dry weight basis, whole grain ingredients exceeds refined grain and meeting accepted standards for healthy foods based on local regulations). Secondary analysis of the National Nutrition and Physical Activity Survey (NNPAS) 2011-2012 dietary intake data included calculation of whole grain intakes based on intake from foods complying with the Healthgrain definition. These were compared with intake values where grams of whole grain in any food had been included.

Setting: Australia.

Participants: Australians ( $\geq 2$ years) who participated in the NNPAS 2011-2012 (n 12 153).

Results: Following expansion of the whole grain database, 214 of the 609 foods containing any amount of whole grain were compliant with the Healthgrain definition. Significant mean differences (all $P<0.05$ ) of $2.84-6.25 \mathrm{~g} / \mathrm{d}$ of whole grain intake (5.91-9.44 g/d energy adjusted) were found when applying the Healthgrain definition in comparison with values from foods containing any whole grain across all age groups.

Conclusions: Application of a whole grain food definition has substantial impact on calculations of population whole grain intakes. While use of such definitions may prove beneficial in settings such as whole grain promotion, the underestimation of total intake may impact on identification of any associations between whole grain intake and health outcomes.
\end{abstract}

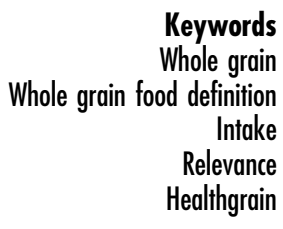

Whole grains are an important part of a healthy diet as an excellent source of protein, carbohydrate, including fibre, and many essential vitamins, minerals, phytochemicals and antioxidants $^{(1)}$. Internationally, it is strongly encouraged that whole grain foods are incorporated daily into the diets of individuals due to their numerous potential health benefits ${ }^{(2-8)}$. Despite extensive epidemiological research on health benefits and international recommendations to consume between 48 and $90 \mathrm{~g}$ whole grain per day, the intake of whole grains globally remains low ${ }^{(9-14)}$.

There are, however, inconsistencies among data regarding the association of whole grain intakes with health outcomes, in particular mortality ${ }^{(15-20)}$, which may be in part explained by variation in the calculation of whole grain intake among studies. Specifically, there are differences in definitions of what contributes to whole grain intake and what constitutes a whole grain food ${ }^{(21,22)}$. For example in the United States, whole grain foods are defined as those containing $\geq 51 \%$ whole grain by weight per reference amount customarily consumed (RACC) by the United States Food and Drug Administration ${ }^{(23)}, \geq 8 \mathrm{~g}$ of whole grain per $30 \mathrm{~g}$ of product by the American Association of Cereal Chemists International (AACCI) ${ }^{(24)}$, while the 2010 Dietary Guidelines for Americans use multiple definitions to classify 
Table 1 Summary of the Healthgrain Forum whole grain food definition*

As outlined by Ross et al. 2017, a whole grain food must contain:

1. $\geq 30 \%$ whole grain ingredients on a dry weight basis and

2. More whole grain than refined grain ingredients

It is strongly advised that whole grain foods meet accepted standards for healthy foods, for example, not being high in sodium, saturated fat and added sugars, based on local regulations. In Australia, foods classified as discretionary are not considered a whole grain food based on this criterion.

Therefore, the Healthgrain whole grain food definition ultimately has three criteria points to classify whole grain foods.

*Definition adapted from Ross et al. (2017) ${ }^{(28)}$.

whole grain foods ${ }^{(25)}$. Therefore notably, some studies include any amount of whole grain from any foods ${ }^{(22,26)}$, some specify a minimum whole grain proportion in foods and only count contributions from those foods ${ }^{(17,19)}$ and some include bran as a whole grain despite not meeting recognised definitions ${ }^{(20,27)}$.

In response, the Healthgrain Forum, an international group of scientists, industry representatives and policymakers advocating for whole grains, have made recommendations for a whole grain food definition that could be applied uniformly across the globe (Table 1$)^{(28)}$. Previously, calculation of whole grain intake in Australia has included grams of whole grain from any source (whether a 'whole grain' food, or a food with some amount of a whole grain ingredient) ${ }^{(9)}$. Using this method, as opposed to more restrictive definitions of whole grain foods, may hold relevance in the context of examining health outcomes associated with whole grain intakes.

Therefore, the aim of the current study was to investigate the impact of using a whole grain food definition on calculated whole grain intake by 1 . determining whole grain intakes in Australian individuals, based on foods that comply with the Healthgrain whole grain food definition and 2. analysing the difference in whole grain intakes when all foods containing any amount of whole grain are included. This also allowed specific analysis of the grams of whole grain which may be contributed from intake of discretionary (higher fat, sugar and salt) foods. The National Nutrition and Physical Activity Survey (NNPAS) 2011-2012 of Australia is used for the current analysis.

\section{Methods}

In brief, the current study investigated the application of the Healthgrain whole grain food definition to the NNPAS 2011-2012 dietary intake data. Results were then compared with whole grain intakes, of the same dietary intake data, when no whole grain food definition was applied. Furthermore, it was of interest to investigate the food sources of whole grain in the sample.
Data and survey population: NNPAS 2011-2012

The NNPAS 2011-2012, a component of the Australian Health Survey, collected data specifically related to health, nutrition and physical activity of 12153 individuals aged 2 years and over. Participants of the survey were usual residents of private dwellings in both rural and urban areas of Australia; however, individuals living in very remote areas were excluded. A complete description of the methods and recruitment of participants are provided elsewhere ${ }^{(29)}$.

\section{Dietary intake data}

The NNPAS 2011-2012 collected dietary intake data of individuals through 24 -h recalls on food and beverages consumed the day prior to the interviews. Interviews were administered by trained Australian Bureau of Statistics (ABS) interviewers over two separate days. The first day of 24-h recalls were conducted through face-to-face interviews with computer assistance ( $n 12153$ ). A second 24-h recall was conducted via telephone within eight days following the first 24-h recall $(n 7735)^{(30)}$. Both 24-h recalls adapted the Automated Multiple-Pass Method (AMPM) to maximise the recall of foods and further limit recall and memory bias. Specific details about the 24 -h recall methods are described elsewhere ${ }^{(29)}$.

\section{Estimation of nutrient-level whole grain intakes}

Estimation of whole grain intakes of survey participants utilised the Australian Food, Supplement and Nutrient Database (AUSNUT) 2011-2013(31). AUSNUT 2011-2013 is compiled of a set of files containing information on various nutrient contents within listed foods, however, whole grain gram values were not included. Therefore, we utilised an Australian whole grain database based on AUSNUT 2011-2013 previously developed by our group ${ }^{(32,33)}$. AUSNUT 2011-2013 was designed specifically with a coding and classification system matched to the foods consumed within the NNPAS, and subsequently nutrient intake estimates of individuals could be calculated. Using the modified version of AUSNUT 2011-2013 with the whole grain values included allowed calculation of whole grain intake. 
Relevance of whole grain food definitions

Table 2 Description of different whole grain food definition approaches applied in analysis to determine whole grain intakes

Approach 1 - No Whole Grain Food Definition

Approach 1a - No whole grain food definition including discretionary foods

- Analysis of all foods containing any amount of whole grain

○ The Healthgrain whole grain food definition is not applied*

Approach $1 \mathrm{~b}-$ No whole grain food definition excluding discretionary foods

- Analysis of all foods containing any amount of whole grain except foods classified as discretionary

- The Healthgrain whole grain food definition is not applied*

- The Australian Health Survey: Users' Guide, 2011-2013 - Discretionary Food List was used to determine discretionary classification $\dagger$

Approach 2 - Application of a Whole Grain Food Definition

Approach $2 \mathrm{a}$ - Application of a whole grain food definition including discretionary foods

$\circ$ Analysis of foods complying with the two main criteria points of the Healthgrain whole grain food definition*

- Foods must contain $\geq 30 \%$ whole grain on a dry weight basis

- Foods must contain more whole grain than refined grain ingredients

'The Healthgrain whole grain food definition refers to the recommendations for a whole grain food definition proposed by Ross et al. (2017).

†The Australian Health Survey: Users' Guide 2011-2013 - Discretionary Food List codes foods in AUSNUT 2011-2013 for discretionary or non-discretionary classification.

Whole grain intakes of survey participants were calculated based on four separate approaches surrounding application of the Healthgrain whole grain food definition to AUSNUT 2011-2013 foods. These approaches hereafter are referred collectively as whole grain food definition approaches and are described in Table 2, which includes approach 1: collation of total whole grain intake in grams (no whole grain food definition), 1a. including discretionary foods, $1 \mathrm{~b}$. excluding discretionary foods; and approach 2: applying the Healthgrain whole grain food definition, $2 \mathrm{a}$. including discretionary foods and $2 \mathrm{~b}$. excluding discretionary foods. The Australian Health Survey: Users' Guide, 2011-2013 - Discretionary Food List ${ }^{(34)}$ was used to identify foods within the AUSNUT database that are deemed discretionary. The Discretionary Food List used various principles to determine discretionary classification $^{(35)}$, including specification and/or inference in the 2013 Australian Dietary Guidelines and supporting documents whereby examples of discretionary foods are listed such as 'most sweet biscuits, cakes, desserts and pastries'. Furthermore, additional criteria based on nutrient profiles were used to determine classification. For grain-based products, in particular, discretionary classification was given to breakfast cereals if sugar content was $>30 \mathrm{~g} /$ $100 \mathrm{~g}$, or $>35 \mathrm{~g} / 100 \mathrm{~g}$ for breakfast cereals with added fruit. For mixed grain-based dishes, discretionary classification was given for those foods with saturated fat $>5 \mathrm{~g} / 100 \mathrm{~g}$. Online Supplementary Material 1 outlines the methods used to determine compliance of AUSNUT foods with the Healthgrain whole grain food definition.

Whole grain intakes were initially calculated on a gram weight per day basis and further adjusted for daily energy intakes to grams per $10 \mathrm{MJ} / \mathrm{d}$, to account for differences in total dietary intakes between age and $\operatorname{sex}^{(10)}$ (online
Supplementary Material 2 - Calculation 1). Whole grain intakes were then analysed for the total participants and further by sex and age for each of the four approaches. Age categories selected for analysis are based on age ranges established for the Australia and New Zealand Nutrient Reference Values (NRVs) for children, adolescents and adults ${ }^{(36)}$. An additional analysis of whole grain intake by age was subsequently completed, combining multiple age groups to form the age categories, hereon referred to as children (2-18 years) and adults (19 years and over).

\section{Multiple Source Method}

For each whole grain food definition approach, usual dietary whole grain intake of participants was calculated using the Multiple Source Method (MSM) (Department of Epidemiology of the German Institute of Human Nutrition Potsdam-Rehbrücke (DIfE), 2008-2011), adjusting data from both $24 \mathrm{~h}$ recalls. The MSM estimates usual dietary intake of nutrients for populations and individuals using a three-step procedure. A more in-depth explanation of the methods behind MSM is discussed elsewhere ${ }^{(37,38)}$.

Prior to analysis through MSM, whole grain intakes of each participant, on each day, were calculated for each whole grain food definition approach. This was achieved through analysis of the NNPAS 2011-2012 Basic Confidentialised Unit Record Files (CURF) data combined with data in the updated Australian whole grain database (see online Supplementary Material 2 - Calculation 2).

Calculated whole grain intakes for each participant, for each day, were run and analysed through MSM using explanatory variables 'age', 'sex' and 'agesex' (age $\times$ sex). A value of 0 was given to 'specify a consumption probability from external source' as consumption frequency was 
irrelevant for the purpose of the current study ${ }^{(38)}$. Usual intake of all participants was used for further analysis. The entire process for MSM was repeated for each whole grain food definition approach.

Dietary energy values were required to compare values of whole grain intake relative to energy intake. Energy intakes for each participant, on each day, were calculated using the energy with fibre values given for each food consumed in the NNPAS Basic CURF dataset. Values were then adjusted through MSM using the same process as described above.

\section{Reporting of food-level whole grain intake (Day 1 only)}

Following the approach outlined in Barrett et al. ${ }^{(39)}$, whole grain intakes at the food level were investigated using unadjusted 'day 1' NNPAS food intake data. The MSM program was not utilised for this step due to an inability to calculate usual intakes of multiple food groups. To report whole grain intake at the food level, the percentage contribution of different food groups to total whole grain intakes was calculated using the 2-digit and 3-digit codes of foods consumed in the NNPAS, aligning with the major and submajor food groups listed within AUSNUT 2011-2013 Food and Dietary Supplement Classification System ${ }^{(40)}$. Two-digit codes represent foods at a category level (e.g. 'cereals and cereal products'), while the 3-digit level further describes foods in these categories (e.g. 'breakfast cereals, ready-to-eat').

Initially, the gram intake of whole grains from any whole grain containing food (approach 1a) was separated into child and adult categories based on consumer age. The total whole grain intake from all participants of each category was then calculated. Foods were then sorted into their respective 2- and 3-digit codes, and total grams of whole grain consumed from each major and sub-major food group were summated. The percentage of total whole grain intake for each major and sub-major food group was separately calculated within each age group category. This process was then repeated for each whole grain food definition approach (approaches 1b, 2a and 2b) (online Supplementary Material 2 - Calculation 3).

\section{Ethics}

Ethics approval was not required as the current study was a secondary analysis of NNPAS 2011-2012 data. Accessibility and dissemination of the ABS Basic CURF data, obtained in the NNPAS, is governed by the Census and Statistics Act $1905^{(41)}$, such that data must remain de-identified. Permission to use and analyse the data were obtained through approved registration at the $\mathrm{ABS}$ registration centre and through the University of Wollongong.

\section{Statistical analyses}

Statistical analysis was performed using the statistical software package IBM SPSS statistics (Statistics version 21,
2009). Data used were positively skewed to the right; however, parametric tests were applied due to the large sample size ( $n$ 12 153). Statistical significance was determined at $P<0.05$ for all analyses.

Median whole grain intakes were determined using the MSM adjusted data of both gram per day and energy adjusted intakes for each of the whole grain food definition approaches. Median and interquartile ranges of intakes were determined for the total participants and further by sex, age and combined sex and age categories. There were ten age categories consisting of $2-3,4-8,9-13,14-18$, 19-30, 31-50, 51-70, >70, 2-18 years (children) and 19 years and over (adults).

Paired samples $t$ test was used to determine if there were significant differences in whole grain intakes between whole grain food definition approaches, but within the same participant categories. For example, intakes of males 2-3 years when no whole grain food definition was applied (including discretionary) were compared with intakes of males 2-3 years when applying a whole grain food definition (including discretionary).

\section{Results}

\section{Compliance of AUSNUT foods}

Following expansion of the whole grain database, 214 of the 609 foods containing any amount of whole grain were compliant with the Healthgrain definition. Online Supplementary Material 1 outlines results in greater detail.

\section{Median whole grain intakes}

Median whole grain intakes of children and adults varied greatly between the whole grain food definition approaches. As expected, the highest whole grain intakes are observed for both children and adults when no whole grain food definition was applied and discretionary foods were included in analysis (approach 1a) (Tables 3 and 4). Lowest whole grain intakes were observed when discretionary foods were excluded with application of a whole grain food definition (approach 2b) (Tables 5 and 6).

Across all whole grain food definition approaches, whole grain intakes, including energy adjusted intakes, were highest among those $>70$ years old. The lowest whole grain intake group varied between the different whole grain food definition approaches and furthermore when adjusting for total energy intake. Lowest whole grain intakes, when adjusted for energy, were seen in the 14-18-year age group for approaches $1 \mathrm{a}$ and $1 \mathrm{~b}$ (Table 4), the 19-30-year age group for approach $2 \mathrm{a}$ (Table 6 ) and the 9-13, 14-18 and 19-30-year age group were equally lowest for approach $2 \mathrm{~b}$ (Table 6 ). This implies that adolescents aged $14-18$ years generally had a low intake of whole grains. This group and the 9-13-year age group consumed more of their relative intake of whole grains from discretionary foods, 
Table 3 Median whole grain intakes ( $g / d)$ for separate participant categories when no whole grain food definition is applied (approach 1)

\begin{tabular}{|c|c|c|c|c|c|c|c|c|c|c|c|c|c|c|c|}
\hline \multirow[b]{3}{*}{ Age (years) ${ }^{\star}$} & \multirow{2}{*}{\multicolumn{3}{|c|}{$n \dagger$}} & \multicolumn{12}{|c|}{ Median whole grain intakes (g/d) } \\
\hline & & & & \multicolumn{4}{|c|}{ Male } & \multicolumn{4}{|c|}{ Female } & \multicolumn{4}{|c|}{ All } \\
\hline & Male & Female & All & $\begin{array}{l}\text { No definition } \\
\text { including } \\
\text { discretionary } \\
\text { (approach 1a) }\end{array}$ & IQR & $\begin{array}{l}\text { No definition } \\
\text { excluding } \\
\text { discretionary } \\
\text { (approach 1b) } \\
\end{array}$ & IQR & $\begin{array}{c}\text { No definition } \\
\text { including } \\
\text { discretionary } \\
\text { (approach 1a) }\end{array}$ & IQR & $\begin{array}{l}\text { No definition } \\
\text { excluding } \\
\text { discretionary } \\
\text { (approach 1b) } \\
\end{array}$ & IQR & $\begin{array}{l}\text { No definition } \\
\text { including } \\
\text { discretionary } \\
\text { (approach 1a) } \\
\end{array}$ & IQR & $\begin{array}{l}\text { No definition } \\
\text { excluding } \\
\text { discretionary } \\
\text { (approach 1b) }\end{array}$ & IQR \\
\hline $2-3$ & 228 & 236 & 464 & 20.54 & $10 \cdot 26-30 \cdot 72$ & 19.47 & $8 \cdot 76-29 \cdot 42$ & 17.81 & $10 \cdot 24-26 \cdot 14$ & $16 \cdot 42$ & $8 \cdot 13-24 \cdot 15$ & $19 \cdot 14$ & $10 \cdot 24-28.73$ & 17.56 & $8.50-27.42$ \\
\hline $4-8$ & 397 & 392 & 789 & $23 \cdot 78$ & $11 \cdot 11-35 \cdot 16$ & 21.68 & $8 \cdot 40-33.44$ & 19.55 & $9 \cdot 49-30 \cdot 21$ & $18 \cdot 13$ & $7.73-28.63$ & 21.96 & $10 \cdot 23-32 \cdot 10$ & 19.65 & $7 \cdot 98-30 \cdot 33$ \\
\hline $9-13$ & 392 & 395 & 787 & $22 \cdot 16$ & $9.09-37.65$ & 18.86 & $0.00-34.99$ & 17.07 & $7.55-27.56$ & 13.58 & $0 \cdot 00-26 \cdot 26$ & 19.77 & $8 \cdot 10-32 \cdot 21$ & $16 \cdot 06$ & $0.00-30.49$ \\
\hline $14-18$ & 403 & 369 & 772 & 21.00 & $0.00-38.13$ & $16 \cdot 04$ & $0.00-36.49$ & 17.45 & $0.00-30.67$ & 14.56 & $0.00-29.03$ & 18.42 & $0.00-34.18$ & $15 \cdot 31$ & $0.00-32.04$ \\
\hline $19-30$ & 739 & 853 & 1592 & 20.97 & $0.00-39.93$ & 18.90 & $0.00-37.81$ & 19.92 & $8 \cdot 71-31 \cdot 32$ & 18.94 & $0.00-30 \cdot 28$ & $20 \cdot 34$ & $6 \cdot 28-35 \cdot 28$ & 18.92 & $0.00-33.14$ \\
\hline $31-50$ & 1669 & 1896 & 3565 & 25.99 & $8 \cdot 70-44.48$ & $25 \cdot 06$ & $0.00-43.12$ & 21.45 & $9.85-34.54$ & 20.44 & $8 \cdot 48-33 \cdot 18$ & 23.33 & $9.45-37.80$ & $22 \cdot 21$ & $0.00-37.23$ \\
\hline $51-70$ & 1341 & 1565 & 2906 & 30.55 & $11.67-47.98$ & 29.97 & $9 \cdot 31-47 \cdot 66$ & $25 \cdot 50$ & $14.22-39.00$ & 24.93 & $13 \cdot 18-38 \cdot 40$ & $27 \cdot 31$ & $13 \cdot 47-43 \cdot 04$ & $26 \cdot 77$ & $12 \cdot 11-42 \cdot 30$ \\
\hline$>70$ & 533 & 745 & 1278 & 33.82 & $19.82-50.28$ & 33.23 & $19.38-50.08$ & 30.77 & $19 \cdot 16-43 \cdot 30$ & 30.48 & $18.71-43.05$ & 31.84 & $19.55-45.93$ & 31.46 & $19.02-45 \cdot 71$ \\
\hline Total children & 1420 & 1392 & 2812 & $22 \cdot 16$ & $9.04-35.59$ & 19.51 & $0.00-33.97$ & 18.08 & $8 \cdot 37-28 \cdot 81$ & $16 \cdot 30$ & $0.00-27.48$ & 19.82 & $8 \cdot 60-32.13$ & 17.53 & $0.00-30.39$ \\
\hline Total adults & 4282 & 5059 & 9341 & 27.86 & $9 \cdot 48-46 \cdot 20$ & $27 \cdot 01$ & $0.00-45.07$ & 24.01 & $11.99-36.75$ & $23 \cdot 11$ & $10.24-35.93$ & 25.52 & $11 \cdot 19-40.51$ & 24.65 & $9.11-39.59$ \\
\hline Total participants & 5702 & 6451 & 12153 & $26 \cdot 18$ & $9 \cdot 25-43 \cdot 72$ & 25.00 & $0.00-42.18$ & 22.50 & $10 \cdot 89-35 \cdot 20$ & $21 \cdot 27$ & $8.93-34.07$ & 24.07 & $10 \cdot 27-38.54$ & 22.92 & $8 \cdot 10-37.55$ \\
\hline
\end{tabular}

*Age categories are those established within the Australia and New Zealand Nutrient Reference Values (NRV). Children are defined as 2-18 year old and adults are defined as 19 year old and over.

†The number of participants refers to the number of individuals that are within that category from the National Nutrition and Physical Activity Survey (NNPAS) 2011-2012. 
Table 4 Energy adjusted median whole grain intakes $(\mathrm{g} / 10 \mathrm{MJ} / \mathrm{d})$ for separate participant categories when no whole grain food definition is applied (approach 1$)$

\begin{tabular}{|c|c|c|c|c|c|c|c|c|c|c|c|c|c|c|c|}
\hline \multirow[b]{3}{*}{ Age $^{*}$} & \multirow{2}{*}{\multicolumn{3}{|c|}{$n \dagger$}} & \multicolumn{12}{|c|}{ Energy adjusted median whole grain intakes $(\mathrm{g} / 10 \mathrm{MJ} / \mathrm{d})$} \\
\hline & & & & \multicolumn{4}{|c|}{ Male } & \multicolumn{4}{|c|}{ Female } & \multicolumn{4}{|c|}{ All } \\
\hline & Male & Female & All & $\begin{array}{l}\text { No definition } \\
\text { including } \\
\text { discretionary } \\
\text { (approach 1a) }\end{array}$ & IQR & $\begin{array}{l}\text { No definition } \\
\text { excluding } \\
\text { discretionary } \\
\text { (approach 1b) }\end{array}$ & IQR & $\begin{array}{l}\text { No definition } \\
\text { including } \\
\text { discretionary } \\
\text { (approach 1a) }\end{array}$ & IQR & $\begin{array}{l}\text { No definition } \\
\text { excluding } \\
\text { discretionary } \\
\text { (approach 1b) }\end{array}$ & IQR & $\begin{array}{l}\text { No definition } \\
\text { including } \\
\text { discretionary } \\
\text { (approach 1a) }\end{array}$ & IQR & $\begin{array}{l}\text { No definition } \\
\text { excluding } \\
\text { discretionary } \\
\text { (approach } 1 \mathrm{~b} \text { ) }\end{array}$ & IQR \\
\hline $2-3$ & 228 & 236 & 464 & $38 \cdot 21$ & $21 \cdot 96-58 \cdot 87$ & 35.73 & $17.92-57.90$ & 37.40 & $22.45-54.56$ & 34.71 & $20 \cdot 31-50.29$ & 38.10 & $22 \cdot 05-55.37$ & 35.29 & $19.44-52.50$ \\
\hline $4-8$ & 397 & 392 & 789 & 37.67 & $18 \cdot 10-60 \cdot 62$ & 35.16 & $13.96-55.52$ & 38.61 & $20.51-59.50$ & $35 \cdot 21$ & $13.01-56.05$ & 37.99 & $19.46-59.99$ & 35.16 & $13.68-55.67$ \\
\hline $9-13$ & 392 & 395 & 787 & $31 \cdot 21$ & $12.43-54.07$ & 25.59 & $0.00-49.69$ & 28.31 & $7 \cdot 86-45 \cdot 71$ & 23.43 & $0.00-43.06$ & $30 \cdot 10$ & $11.51-49.85$ & 24.54 & $0.00-47 \cdot 14$ \\
\hline $14-18$ & 403 & 369 & 772 & 26.44 & $0.00-49.45$ & 20.59 & $0.00-46.47$ & 28.94 & $0.00-51.26$ & $24 \cdot 27$ & $0.00-48.97$ & 28.01 & $0.00-50.33$ & 23.29 & $0.00-47.47$ \\
\hline $19-30$ & 739 & 853 & 1592 & 27.04 & $0.00-49.67$ & 24.08 & $0.00-47.60$ & 32.79 & $12 \cdot 78-53.29$ & 30.77 & $0.00-50.92$ & 30.48 & $6.03-52.08$ & 27.79 & $0.00-49.95$ \\
\hline $31-50$ & 1669 & 1896 & 3565 & 34.35 & $11 \cdot 16-56.95$ & 32.56 & $0.00-55.89$ & 36.59 & $16.36-56.64$ & 33.86 & $11 \cdot 36-55.26$ & 35.14 & $13.56-56.76$ & 33.46 & $0.00-55.41$ \\
\hline $51-70$ & 1341 & 1565 & 2906 & $41 \cdot 26$ & $15.54-65.01$ & 40.68 & $13 \cdot 46-64 \cdot 66$ & 43.84 & $24 \cdot 72-65 \cdot 77$ & 42.56 & $22.90-64.57$ & 42.53 & $21 \cdot 20-65 \cdot 33$ & 41.77 & $19.35-64.56$ \\
\hline$>70$ & 533 & 745 & 1278 & 49.58 & $27.93-70.63$ & $48 \cdot 23$ & $27 \cdot 33-70 \cdot 35$ & 56.08 & $35 \cdot 60-75 \cdot 80$ & 55.59 & $34 \cdot 28-75 \cdot 31$ & 53.53 & $32 \cdot 30-73 \cdot 78$ & 52.91 & $31.49-73.46$ \\
\hline Total children & 1420 & 1392 & 2812 & 33.94 & $13.71-54.76$ & $30 \cdot 23$ & $0.00-51.37$ & 32.96 & $14 \cdot 12-52.84$ & 29.64 & $0.00-49.69$ & 33.28 & $13.96-53.62$ & 29.98 & $0.00-50.67$ \\
\hline Total adults & 4282 & 5059 & 9341 & 37.36 & $12 \cdot 50-61 \cdot 14$ & $36 \cdot 31$ & $0.00-60.23$ & 41.07 & $20 \cdot 05-62.77$ & 39.43 & $17 \cdot 38-61 \cdot 60$ & 39.35 & $17 \cdot 03-62 \cdot 15$ & 38.23 & $13.23-60.97$ \\
\hline Total participants & 5702 & 6451 & 12153 & 38.42 & $16.40-60.90$ & 36.35 & $11 \cdot 29-59 \cdot 15$ & 37.94 & $15 \cdot 88-60 \cdot 16$ & 36.26 & $11.08-58.86$ & 38.16 & $16 \cdot 18-60.52$ & $36 \cdot 27$ & $11 \cdot 15-58.94$ \\
\hline
\end{tabular}

*Age categories are those established within the Australia and New Zealand Nutrient Reference Values (NRV). Children are defined as 2-18 year old and adults are defined as 19 year old and over.

tThe number of participants refers to the number of individuals that are within that category from the National Nutrition and Physical Activity Survey (NNPAS) $2011-2012$. 
Table 5 Median whole grain intakes (g/d) for separate participant categories when applying the Healthgrain whole grain food definition (approach 2)

\begin{tabular}{|c|c|c|c|c|c|c|c|c|c|c|c|c|c|c|c|}
\hline \multirow[b]{3}{*}{ Age (years) ${ }^{*}$} & \multirow{2}{*}{\multicolumn{3}{|c|}{$n \dagger$}} & \multicolumn{12}{|c|}{ Median whole grain intakes $(\mathrm{g} / \mathrm{d})$} \\
\hline & & & & \multicolumn{4}{|c|}{ Male } & \multicolumn{4}{|c|}{ Female } & \multicolumn{4}{|c|}{ All } \\
\hline & Male & Female & All & $\begin{array}{l}\text { Definition } \\
\text { including } \\
\text { discretionary } \\
\text { (approach 2a) }\end{array}$ & IQR & $\begin{array}{l}\text { Definition } \\
\text { excluding } \\
\text { discretionary } \\
\text { (approach } 2 b \text { ) }\end{array}$ & IQR & $\begin{array}{l}\text { Definition } \\
\text { including } \\
\text { discretionary } \\
\text { (approach 2a) }\end{array}$ & IQR & $\begin{array}{l}\text { Definition } \\
\text { excluding } \\
\text { discretionary } \\
\text { (approach } 2 \mathrm{~b} \text { ) }\end{array}$ & IQR & $\begin{array}{c}\text { Definition } \\
\text { including } \\
\text { discretionary } \\
\text { (approach 2a) }\end{array}$ & IQR & $\begin{array}{l}\text { Definition } \\
\text { excluding } \\
\text { discretionary } \\
\text { (approach 2b) }\end{array}$ & IQR \\
\hline $2-3$ & 228 & 236 & 464 & 18.51 & $0.00-28.25$ & 18.62 & $0.00-28 \cdot 18$ & 16.87 & $0.00-24.95$ & 16.00 & $0.00-24.83$ & 17.65 & $0.00-26.93$ & 17.02 & $0.00-26.78$ \\
\hline $4-8$ & 397 & 392 & 789 & 21.61 & $0.00-33.15$ & $20 \cdot 21$ & $0.00-31.70$ & $18 \cdot 11$ & $0.00-28.11$ & 17.38 & $0.00-27.71$ & 19.51 & $0.00-31 \cdot 10$ & 18.50 & $0.00-30.43$ \\
\hline $9-13$ & 392 & 395 & 787 & 19.73 & $0.00-35.38$ & 14.48 & $0.00-34.68$ & 14.90 & $0.00-25.73$ & 0.00 & $0.00-24.87$ & 17.39 & $0.00-31.26$ & 0.00 & $0.00-30.83$ \\
\hline $14-18$ & 403 & 369 & 772 & $12 \cdot 81$ & $0.00-38.08$ & 0.00 & $0.00-35.58$ & 15.02 & $0.00-30.41$ & 0.00 & $0.00-29.00$ & $14 \cdot 22$ & $0.00-32.86$ & 0.00 & $0.00-32.29$ \\
\hline $19-30$ & 739 & 853 & 1592 & 0.00 & $0.00-39.01$ & 0.00 & $0.00-37.47$ & $15 \cdot 62$ & $0.00-29.84$ & 0.00 & $0.00-29.65$ & 14.49 & $0.00-33.72$ & 0.00 & $0.00-33.17$ \\
\hline $31-50$ & 1669 & 1896 & 3565 & $21 \cdot 13$ & $0.00-42.47$ & $19 \cdot 21$ & $0.00-42 \cdot 21$ & $18 \cdot 62$ & $0.00-32.58$ & 16.64 & $0.00-32.53$ & 19.58 & $0.00-36.10$ & $17 \cdot 30$ & $0.00-36.14$ \\
\hline $51-70$ & 1341 & 1565 & 2906 & 27.55 & $0.00-45.45$ & $27 \cdot 41$ & $0.00-45 \cdot 10$ & 23.07 & $0.00-36.87$ & $22 \cdot 72$ & $0.00-36.74$ & 24.74 & $0.00-39.82$ & 24.37 & $0.00-39.77$ \\
\hline$>70$ & 533 & 745 & 1278 & 30.00 & $0.00-47.22$ & 30.00 & $0.00-46.12$ & 26.97 & $14.02-39.75$ & $26 \cdot 28$ & $12.54-39.63$ & 28.29 & $0.00-42.32$ & 27.92 & $0.00-42 \cdot 12$ \\
\hline Total children & 1420 & 1392 & 2812 & $19 \cdot 34$ & $0.00-34.38$ & 16.74 & $0.00-33.06$ & 16.57 & $0.00-27.69$ & 13.90 & $0.00-27.48$ & 17.73 & $0.00-30.88$ & 15.08 & $0.00-30.26$ \\
\hline Total adults & 4282 & 5059 & 9341 & $24 \cdot 10$ & $0.00-43.67$ & 23.42 & $0.00-43.12$ & $21 \cdot 24$ & $0.00-34.43$ & 20.58 & $0.00-34 \cdot 21$ & $22 \cdot 27$ & $0.00-37.98$ & 21.47 & $0.00-37.68$ \\
\hline Total participants & 5702 & 6451 & 12153 & 22.39 & $0.00-41.07$ & 21.02 & $0.00-40.65$ & 19.76 & $0.00-33.16$ & 18.85 & $0.00-32.90$ & 20.73 & $0.00-36.45$ & 19.55 & $0.00-35.98$ \\
\hline
\end{tabular}

${ }^{*}$ Age categories are those established within the Australia and New Zealand Nutrient Reference Values (NRV). Children are defined as 2-18 year old and adults are defined as 19 year old and over.

tThe number of participants refers to the number of individuals that are within that category from the National Nutrition and Physical Activity Survey (NNPAS) $2011-2012$. 
Table 6 Energy adjusted median whole grain intakes (g/10 MJ/d) for separate participant categories when applying the Healthgrain whole grain food definition (approach 2)

\begin{tabular}{|c|c|c|c|c|c|c|c|c|c|c|c|c|c|c|c|}
\hline \multirow[b]{3}{*}{ Age (years) ${ }^{*}$} & \multirow{2}{*}{\multicolumn{3}{|c|}{$n \dagger$}} & \multicolumn{12}{|c|}{ Energy adjusted median whole grain intakes $(\mathrm{g} / 10 \mathrm{MJ} / \mathrm{d})$} \\
\hline & & & & \multicolumn{4}{|c|}{ Male } & \multicolumn{4}{|c|}{ Female } & \multicolumn{4}{|c|}{ All } \\
\hline & Male & Female & All & $\begin{array}{c}\text { Definition } \\
\text { including } \\
\text { discretionary } \\
\text { (approach 2a) } \\
\end{array}$ & IQR & $\begin{array}{c}\text { Definition } \\
\text { excluding } \\
\text { discretionary } \\
\text { (approach } 2 \mathrm{~b} \text { ) }\end{array}$ & IQR & $\begin{array}{c}\text { Definition } \\
\text { including } \\
\text { discretionary } \\
\text { (approach 2a) }\end{array}$ & IQR & $\begin{array}{c}\text { Definition } \\
\text { excluding } \\
\text { discretionary } \\
\text { (approach } 2 \mathrm{~b} \text { ) }\end{array}$ & IQR & $\begin{array}{c}\text { Definition } \\
\text { including } \\
\text { discretionary } \\
\text { (approach 2a) }\end{array}$ & IQR & $\begin{array}{c}\text { Definition } \\
\text { excluding } \\
\text { discretionary } \\
\text { (approach } 2 \mathrm{~b} \text { ) }\end{array}$ & IQR \\
\hline $2-3$ & 228 & 236 & 464 & 33.55 & $0.00-52.78$ & 33.26 & $0.00-52 \cdot 76$ & $35 \cdot 30$ & $0.00-51.69$ & 33.79 & $0.00-50.00$ & 34.68 & $0.00-51.96$ & 33.35 & $0.00-51 \cdot 12$ \\
\hline $4-8$ & 397 & 392 & 789 & 34.12 & $0.00-54.98$ & 32.41 & $0.00-52.45$ & 34.95 & $0.00-55.64$ & 33.28 & $0.00-54.40$ & 34.44 & $0.00-55.21$ & 32.64 & $0.00-53.47$ \\
\hline $9-13$ & 392 & 395 & 787 & $28 \cdot 71$ & $0.00-50 \cdot 15$ & 19.43 & $0.00-48.99$ & 25.69 & $0.00-42.76$ & 0.00 & $0.00-42.48$ & 26.63 & $0 \cdot 00-46 \cdot 50$ & 0.00 & $0 \cdot 00-45 \cdot 21$ \\
\hline $14-18$ & 403 & 369 & 772 & $15 \cdot 62$ & $0.00-50 \cdot 70$ & 0.00 & $0.00-46.91$ & 24.67 & $0.00-51.50$ & 0.00 & $0.00-50.94$ & 20.85 & $0.00-50.86$ & 0.00 & $0.00-48.65$ \\
\hline $19-30$ & 739 & 853 & 1592 & 0.00 & $0.00-51.27$ & 0.00 & $0.00-49 \cdot 33$ & 24.78 & $0.00-48.78$ & 0.00 & $0.00-48.15$ & 19.85 & $0.00-49.66$ & 0.00 & $0.00-48.65$ \\
\hline $31-50$ & 1669 & 1896 & 3565 & 26.96 & $0.00-53.51$ & 23.29 & $0.00-53.42$ & 29.95 & $0.00-54.34$ & 27.06 & $0.00-54.01$ & 28.71 & $0.00-54.00$ & 25.64 & $0.00-53.86$ \\
\hline $51-70$ & 1341 & 1565 & 2906 & 37.47 & $0.00-61.28$ & 37.23 & $0.00-61.46$ & 39.57 & $0.00-61 \cdot 27$ & 38.78 & $0.00-60.65$ & 38.56 & $0.00-61.22$ & 38.07 & $0.00-61 \cdot 13$ \\
\hline$>70$ & 533 & 745 & 1278 & 43.82 & $0.00-66.37$ & 43.42 & $0.00-66.56$ & 48.91 & $22.61-70.97$ & 48.52 & $19 \cdot 25-71 \cdot 18$ & 45.91 & $0.00-68.71$ & 45.53 & $0.00-68.80$ \\
\hline Total children & 1420 & 1392 & 2812 & 30.42 & $0.00-51.91$ & 26.05 & $0.00-50.43$ & $30 \cdot 20$ & $0.00-49.87$ & 26.92 & $0.00-48.83$ & $30 \cdot 29$ & $0.00-50.75$ & $26 \cdot 66$ & $0.00-49.40$ \\
\hline Total adults & 4282 & 5059 & 9341 & 31.79 & $0.00-58.18$ & 30.46 & $0.00-57.90$ & 35.65 & $0.00-58 \cdot 27$ & 34.08 & $0.00-57.92$ & 34.08 & $0.00-58 \cdot 20$ & $32 \cdot 61$ & $0.00-57.90$ \\
\hline Total participants & 5702 & 6451 & 12153 & 33.31 & $0.00-56.90$ & 31.59 & $0.00-56.39$ & $32 \cdot 23$ & $0.00-56.37$ & 30.35 & $0.00-55.95$ & 32.80 & $0.00-56.66$ & 31.00 & $0.00-56.14$ \\
\hline
\end{tabular}

*Age categories are those established within the Australia and New Zealand Nutrient Reference Values (NRV). Children are defined as 2-18 year old and adults are defined as 19 year old and over.

†The number of participants refers to the number of individuals that are within that category from the National Nutrition and Physical Activity Survey (NNPAS) $2011-2012$. 
and 19-30-year-olds consumed whole grains from foods that do not comply with the Healthgrain definition, namely, those containing significant refined grains. There were minimal differences in energy adjusted whole grain intakes between males and females among each of the whole grain food definition approaches.

\section{Comparison of whole grain intakes between whole grain food definition approaches}

Statistically significant differences $(P<0.05)$ exist between whole grain intake values of different whole grain food definition approaches within all participant categories (Table 7). The difference in mean whole grain intake ranged from 2.09 to $5.12 \mathrm{~g} / \mathrm{d}(3.77-8.66 \mathrm{~g} / 10 \mathrm{MJ} / \mathrm{d})$ across participant categories when comparing approach 1a to approach $2 \mathrm{a}$ (Table 7). Mean difference in whole grain intakes when comparing approach $1 \mathrm{a}$ to approach $2 \mathrm{~b}$ and approach $1 \mathrm{~b}$ to approach $2 \mathrm{~b}$ ranged from 2.84 to $6.25 \mathrm{~g} / \mathrm{d}(5.91-9.44 \mathrm{~g} / 10 \mathrm{MJ} / \mathrm{d})$ and $1.26-4.81 \mathrm{~g} / \mathrm{d}(2.57-$ $8.28 \mathrm{~g} / 10 \mathrm{MJ} / \mathrm{d}$ ) across participant categories, respectively.

\section{Food sources of whole grain intakes (Day 1 only)}

Calculating the percentage of total whole grain intake of major and sub-major food groups for both children and adults found that, for all whole grain food definition approaches, the highest percentage of whole grain intakes was from the 'cereals and cereal products' major food group. The total intake percentage for this food group was mostly higher for adults in comparison with children within the same whole grain food definition approach, with the exception of approach $2 \mathrm{~b}$ (Table 8 and online Supplemental Tables S4 and S5).

When comparing total percentage intakes across the whole grain food definition approaches, there were major differences in the 'cereal and cereal products', 'snack foods' and 'confectionary and cereal/nut/fruit/seed bars' major food groups for both children and adults (Table 8). These differences can be attributed to the exclusion of whole grain within specific foods when applying the Healthgrain definition, particularly discretionary items including corn chips, popcorn and muesli or cereal style bars. In terms of relative percentage contribution, this is most relevant in children who gain almost $10 \%$ of their total whole grain intake from these discretionary items.

\section{Discussion}

The current study identifies two key findings within the research of grains and the translation of messages around whole grain intakes. First, these results suggest that the way in which whole grain intakes are calculated provides significant variation in measured whole grain intake, with potential relevance to investigating associated health benefits. Second, specific types of foods containing whole grains may be excluded from intake calculations due to other components such as refined grains or having higher sugar, saturated fat or sodium content, yet it is difficult to truly ascertain if these potentially deleterious components negate the effect of the whole grain within the food. Both these considerations have the potential to affect how organisations and individual health professionals promote increases in whole grain intake.

Current research suggests that utilising a unified whole grain food definition will have positive outcomes in whole grain promotion and subsequently in increasing population whole grain intakes, such that it may influence whole grain product labelling and further assist consumers to choose healthier and higher whole grain content products $^{(21,28)}$. However, acceptance of a whole grain food definition by consumers and their organisations is essential to achieve adequate whole grain promotion. On the contrary, there are potential implications when applying a definition in research investigating the impacts of whole grain intakes on health. Here, and elsewhere ${ }^{(21)}$, whole grain containing food products that do not comply with the whole grain food definition and are excluded from analysis may still contribute to an individual's whole grain intake. Failure to account for these products can significantly impact calculated whole grain intakes, and therefore impact study outcomes using this data, specifically affecting the observed associations with health outcomes ${ }^{(21)}$.

Similarly to our work, a study by Mann et $a l^{(10)}$ on UK data found that whole grain intakes were lower when applying different criteria to define whole grain foods, such that $\mathrm{a} \geq 10 \%$ and $\mathrm{a} \geq 51 \%$ whole grain proportion cut-off was applied in comparison with absolute whole grain intakes. Similarly, another study recognised that applying various criteria to define whole grain foods has the ability to underestimate whole grain intakes of populations, especially utilising definitions whereby foods must contain a high proportion of whole grains ${ }^{(42)}$. Thus, if the Healthgrain whole grain food definition is to be applied and utilised in future research, we, as an international community, must acknowledge and further recognise that values given do not truly reflect individual and population whole grain intakes. It is evident in the present study that applying a whole grain food definition, especially incorporating discretionary food exclusion, create differences in reported whole grain intake values of potential clinical relevance.

In general, exclusion of discretionary foods from the current analysis, whether a whole grain food definition is applied or not, caused reductions in whole grain intake values, which was most evident among the older children and adolescent age groups. This is likely due to greater discretionary food intakes in general, with current research in the Australian context highlighting discretionary food consumption is highest among the 14-18-year age group ${ }^{(43)}$. Furthermore, the proportion of energy from discretionary foods is shown to decrease in the 19-30-year age group 
Table 7 Difference in mean whole grain intake between different whole grain food definition approaches

\begin{tabular}{|c|c|c|c|c|c|c|c|c|c|}
\hline \multirow[b]{3}{*}{ Age groups†‡ } & \multicolumn{9}{|c|}{ Difference in mean whole grain consumption $(\mathrm{g} / \mathrm{d}) \S$} \\
\hline & \multicolumn{3}{|c|}{$\begin{array}{l}\text { No definition including discretionary } \\
\text { (approach 1a) and definition including } \\
\text { discretionary (approach 2a) }\end{array}$} & \multicolumn{3}{|c|}{$\begin{array}{l}\text { No definition including discretionary } \\
\text { (approach 1a) and definition excluding } \\
\text { discretionary (approach } 2 b \text { ) }\end{array}$} & \multicolumn{3}{|c|}{$\begin{array}{l}\text { No definition excluding discretionary } \\
\text { (approach } 1 \mathrm{~b} \text { ) and definition excluding } \\
\text { discretionary (approach } 2 \mathrm{~b} \text { ) }\end{array}$} \\
\hline & Male & Female & All & Male & Female & All & Male & Female & All \\
\hline $2-3$ years & 3.34 & 2.09 & $2 \cdot 70$ & 3.77 & $2 \cdot 84$ & $3 \cdot 30$ & 2.52 & 1.26 & 1.88 \\
\hline Energy adjusted & 6.40 & 4.28 & 5.33 & 6.96 & 5.91 & 6.43 & 4.77 & 2.57 & 3.65 \\
\hline $4-8$ years & $2 \cdot 82$ & $3 \cdot 20$ & 3.01 & 4.58 & $4 \cdot 27$ & 4.42 & $2 \cdot 61$ & $2 \cdot 26$ & 2.44 \\
\hline Energy adjusted & 4.67 & $6 \cdot 17$ & 5.42 & $7 \cdot 50$ & $8 \cdot 11$ & $7 \cdot 80$ & 4.34 & 4.37 & 4.35 \\
\hline $9-13$ years & 3.83 & 3.37 & 3.59 & $5 \cdot 85$ & $5 \cdot 22$ & 5.53 & 2.63 & 2.97 & $2 \cdot 80$ \\
\hline Energy adjusted & 5.33 & 5.45 & 5.39 & 8.07 & 8.59 & 8.33 & 3.63 & 4.96 & 4.30 \\
\hline $14-18$ years & 4.28 & $2 \cdot 35$ & 3.36 & $6 \cdot 25$ & $4 \cdot 21$ & $5 \cdot 27$ & 3.73 & $2 \cdot 37$ & 3.08 \\
\hline Energy adjusted & $5 \cdot 15$ & 3.77 & 4.49 & 7.91 & 6.93 & 7.44 & 4.62 & 3.82 & $4 \cdot 24$ \\
\hline $19-30$ years & 3.97 & 4.52 & 4.27 & 5.58 & 5.72 & $5 \cdot 66$ & 3.80 & $4 \cdot 11$ & 3.97 \\
\hline Energy adjusted & 4.79 & 7.54 & $6 \cdot 27$ & $6 \cdot 80$ & 9.44 & $8 \cdot 21$ & 4.58 & $6 \cdot 87$ & $5 \cdot 81$ \\
\hline $31-50$ years & 4.72 & 4.01 & 4.34 & 5.54 & 4.79 & $5 \cdot 14$ & 4.25 & 3.65 & 3.93 \\
\hline Energy adjusted & 6.06 & 6.49 & $6 \cdot 29$ & 7.14 & $7 \cdot 79$ & 7.49 & 5.51 & 5.90 & $5 \cdot 72$ \\
\hline $51-70$ years & 4.85 & 4.59 & 4.71 & 5.09 & 4.89 & 4.98 & 4.56 & $4 \cdot 14$ & 4.33 \\
\hline Energy adjusted & 6.40 & $7 \cdot 76$ & $7 \cdot 13$ & $6 \cdot 70$ & $8 \cdot 30$ & 7.56 & 5.97 & 7.03 & 6.54 \\
\hline$>70$ years & $5 \cdot 12$ & 4.87 & 4.98 & $5 \cdot 34$ & $5 \cdot 14$ & $5 \cdot 22$ & 4.81 & 4.66 & 4.72 \\
\hline Energy adjusted & 6.95 & 8.66 & 7.95 & $7 \cdot 37$ & 9.05 & 8.35 & 6.54 & 8.28 & 7.56 \\
\hline Total children & 3.60 & $2 \cdot 83$ & 3.22 & $5 \cdot 27$ & 4.28 & 4.78 & 2.92 & $2 \cdot 32$ & $2 \cdot 62$ \\
\hline Energy adjusted & $5 \cdot 27$ & 5.01 & $5 \cdot 14$ & 7.69 & 7.56 & 7.62 & 4.29 & 4.09 & 4.19 \\
\hline Total adults & 4.68 & 4.40 & 4.53 & $5 \cdot 38$ & 5.03 & $5 \cdot 19$ & 4.34 & 4.03 & $4 \cdot 17$ \\
\hline Energy adjusted & 6.06 & 7.38 & 6.77 & 6.97 & 8.41 & $7 \cdot 75$ & $5 \cdot 62$ & 6.76 & $6 \cdot 24$ \\
\hline Total participants & 4.41 & 4.06 & 4.23 & $5 \cdot 36$ & 4.87 & $5 \cdot 10$ & 3.97 & 3.66 & 3.81 \\
\hline Energy adjusted & $5 \cdot 86$ & 6.87 & $6 \cdot 40$ & $7 \cdot 15$ & 8.23 & $7 \cdot 72$ & $5 \cdot 29$ & $6 \cdot 19$ & $5 \cdot 77$ \\
\hline
\end{tabular}

${ }^{*}$ There is a statistically significant difference among all comparisons $(P<0.05)$.

tAge groups by years are in line with those within the Australia and New Zealand Nutrient Reference Values (NRVs).

tAge groups by children and adult are defined as children $=2-18$ years and adult $=19$ year old and over

$\S$ Difference in means obtained through paired samples $t$ test ( $t$ test for equality of means). 
Table 8 Percentages of total whole grain intakes for major food groups from each whole grain food definition approach

\begin{tabular}{|c|c|c|c|c|c|c|c|c|}
\hline \multirow[b]{2}{*}{ Major food group* } & \multicolumn{2}{|c|}{$\begin{array}{l}\text { No definition including } \\
\text { discretionary } \\
\text { (approach 1a) }\end{array}$} & \multicolumn{2}{|c|}{$\begin{array}{l}\text { No definition } \\
\text { excluding } \\
\text { discretionary } \\
\text { (approach } 1 b \text { ) }\end{array}$} & \multicolumn{2}{|c|}{$\begin{array}{l}\text { Definition including } \\
\text { discretionary } \\
\text { (approach 2a) }\end{array}$} & \multicolumn{2}{|c|}{$\begin{array}{l}\text { Definition excluding } \\
\text { discretionary } \\
\text { (approach } 2 \mathrm{~b} \text { ) }\end{array}$} \\
\hline & $\begin{array}{l}\text { Children† } \\
(\%)\end{array}$ & $\begin{array}{l}\text { Adults } \neq \\
(\%)\end{array}$ & $\begin{array}{l}\text { Children } \\
(\%)\end{array}$ & $\begin{array}{l}\text { Adults } \\
(\%)\end{array}$ & $\begin{array}{l}\text { Children } \\
(\%)\end{array}$ & $\begin{array}{l}\text { Adults } \\
(\%)\end{array}$ & $\begin{array}{l}\text { Children } \\
(\%)\end{array}$ & $\begin{array}{l}\text { Adults } \\
(\%)\end{array}$ \\
\hline Cereal and cereal products & 82.97 & 89.56 & $92 \cdot 64$ & 93.07 & $86 \cdot 58$ & 92.68 & $95 \cdot 85$ & $95 \cdot 77$ \\
\hline Cereal-based products and dishes & $6 \cdot 13$ & 6.50 & 6.08 & 5.93 & 2.63 & $3 \cdot 21$ & 2.91 & 3.32 \\
\hline Snack foods & 4.89 & $1 \cdot 31$ & 0.49 & 0.09 & 5.48 & 1.55 & 0.57 & 0.11 \\
\hline $\begin{array}{l}\text { Confectionary and cereal/nut/fruit/ } \\
\text { seed bars }\end{array}$ & 4.50 & 1.75 & 0.00 & 0.00 & $4 \cdot 70$ & 1.79 & 0.00 & 0.00 \\
\hline
\end{tabular}

*Major food groups are those described in AUSNUT 2011-2013 Food and Dietary Supplement Classification System using the 2-digit food code. †Children $=2-18$ year old.

$\ddagger$ Adult $=19$ year old and over.

and older ${ }^{(43)}$. These differences are related to a multitude of factors, however, can be associated with specific lifestyle factors and other influences surrounding individuals at specific life stages ${ }^{(44)}$. The high prevalence of discretionary food consumption has negative consequences on calculated whole grain intake. Due to a higher demand, manufacturers are more inclined to add whole grains to these food items, potentially improving their healthful properties. However, it may be less than ideal to encourage a discretionary food, regardless of whole grain content, particularly if it is a small amount.

In the current study, a number of whole grain containing foods excluded due to non-compliance with the definition contained substantial amounts of whole grain. From the current analysis, it is clear in approaches where a whole grain food definition is applied, the percentages of total whole grain intake shows little to no whole grains being consumed from major food groups other than the 'Cereals and cereal products' or 'Cereal based products and dishes' major food groups. However, a larger variation of whole grain food sources are shown where no whole grain food definition was applied, thus illustrating impact on the distribution of total whole grain intake food sources, when a whole grain food definition is applied.

Moreover, as shown through intake comparisons, decreases of up to $8 \mathrm{~g}$ of whole grain (energy adjusted) were found when applying the Healthgrain definition. Eight grams is considered half a whole grain serve and is potentially of clinical relevance. Studies have found that one single whole grain serving $(16 \mathrm{~g})$ can reduce fasting insulin concentrations by $6.3 \%{ }^{(45)}$ and reduce weight gain $^{(46)}$. Additionally, one whole grain food serving (30 g with around $16 \mathrm{~g}$ whole grain) has been reported to reduce all-cause mortality by $7 \%$ and CVD-specific mortality by $8 \%{ }^{(47)}$. The undercalculating related to application of food definitions is therefore a key consideration for future work.

Limitations here relate to the data sources used for analysis with the retrospective analysis of the NNPAS 2011-2012 unable to represent the 'current' whole grain consumption of Australians. The current analysis did not apply replicate weights to account for the sampling design of the Australian Health Survey. While appropriate given this is a methodological study exploring the impact of different whole grain definitions, caution should be applied when generalising these findings to national whole grain intakes. Additionally, since collection of the NNPAS data, there has been an increase in whole grain products introduced into the Australian market, including development of new products and enrichment of existing products, while some whole grain products have been removed ${ }^{(48)}$ introducing further potential error. This is however the most recent and comprehensive dataset available for Australian intake data and, especially as this is a comparative methods study, can be used as guidance to provide a general estimation of whole grain intakes among individuals.

In the current study, whole grain intakes were calculated based on inclusion of all participants, regardless if they were a whole grain consumer or non-consumer. Although these intake values provide an overview of whole grain consumption among survey participants, they may not hold relevance when conducting further analysis or comparisons due to estimation based on large proportions of non-consumers. While it may have been beneficial, in the current study, to conduct separate analysis on consumers only, a study by Galea et al. (2017) ${ }^{(9)}$ has previously analysed consumer only whole grain intakes using the same NNPAS dietary intake data. It was found almost $30 \%$ of all participants were non-consumers of whole grains, such that median whole grain intake in adults increased by $17 \mathrm{~g} / \mathrm{d}$ when analysing consumers only. This provides an adequate indication for the percentage of whole grain consumers and nonconsumers in the Australian context; however, methods used to calculate whole grain intake differ slightly to the present study.

Additionally, findings from the study cannot be implied internationally since dietary intake data vary from country to country, as does the food available to consumers and their composition. However, the methods used in the current study may provide guidance for future studies and may act as a comparative study to the present research. 
Assumptions in the whole grain food database, regarding food compliance with the Healthgrain definition, are underlying throughout intake calculations and have the potential to over or underestimate whole grain intake values. The data within the whole grain database may also not reflect true whole grain composition of foods as values are derived from ingredient labels, estimated recipes and data obtained elsewhere. Therefore, variations within the same product and changes in formulations cannot be accounted for and may alter total whole grain intake values. Nonetheless, the limitations present within this current study are similar to those within other studies alike ${ }^{(9-11,49)}$.

It is evident that application of a whole grain food definition, namely, the definition proposed by the Healthgrain Forum, has potentially significant impacts on measurement of whole grain intakes of individuals and populations. While application of a whole grain food definition may prove beneficial within settings such as whole grain promotion, where individuals will understand to eat more of a food rather than to eat ' $x$ more grams of whole grain', future research must also investigate the potential implications this has among other settings, namely, the associations between whole grain intakes and health outcomes. The two settings are different, and careful differentiation is essential in both whole grain health research and whole grain promotion.

\section{Acknowledgements}

Acknowledgements: The authors thank S.G. for guidance surrounding whole grain food products and definitions in the Australian context and E.B. for assistance with multiple source method (MSM) data analysis. Financial support: This research received no specific grant from any funding agency, commercial or not-for-profit sectors. Conflict of interest: There are no conflicts of interest. Authorship: K.R.K. and E.J.B. formulated the research question; K.R.K. and E.J.B. designed the study; K.R.K. conducted research; K.R.K. and E.P.N. analysed data; K.R.K., E.P.N. and E.J.B. wrote the article; K.R.K. had primary responsibility for final content. All authors read and approved the final manuscript. Ethics of buman subject participation: Ethics approval was not required for this current study. Accessibility and dissemination of data in the Australian Bureau of Statistics (ABS) Basic Confidentialised Unit Record Files (CURF) is governed by the Census and Statistics Act 1905, such that data must remain de-identified. Permission to use and analyse the data were obtained through approved registration at the ABS registration centre and through the University of Wollongong.

Census and Statistics Act available at: https://www. legislation.gov.au/Details/C2016C01005

\section{Supplementary material}

For supplementary material accompanying this paper visit https://doi.org/10.1017/S1368980019004452

\section{References}

1. State Government of Victoria (2012) Cereals and Wholegrain Foods. Victoria, Australia: Department of Health and Human Services.

2. Chen G, Tong X, Xu JY et al. (2016) Whole-grain intake and total, cardiovascular, and cancer mortality: a systematic review and meta-analysis of prospective studies. Am J Clin Nutr 104, 164-172.

3. Zhang B, Zhao Q, Guo W et al. (2018) Association of whole grain intake with all-cause, cardiovascular, and cancer mortality: a systematic review and dose-response metaanalysis from prospective cohort studies. Eur J Clin Nutr 72, 57-65.

4. Ye EQ, Chacko SA, Chou EL et al. (2012) Greater whole-grain intake is associated with lower risk of type 2 diabetes, cardiovascular disease, and weight gain. J Nutr 142, 1304-1313.

5. Aune D, Norat T, Romundstad P et al. (2013) Whole grain and refined grain consumption and the risk of type 2 diabetes: a systematic review and dose-response meta-analysis of cohort studies. Eur J Epidemiol 28, 845-858.

6. Aune D, Chan DSM, Lau R et al. (2011) Dietary fibre, whole grains, and risk of colorectal cancer: systematic review and dose-response meta-analysis of prospective studies. BMJ 343, d6617. Published online: 10 November 2011. doi: 10. 1136/bmj.d6617.

7. Harland JI \& Garton LE (2008) Whole-grain intake as a marker of healthy body weight and adiposity. Public Health Nutr 11, 554-563.

8. Newby PK, Maras J, Bakun P et al. (2007) Intake of whole grains, refined grains, and cereal fiber measured with 7-d diet records and associations with risk factors for chronic disease. Am J Clin Nutr 86, 1745-1753.

9. Galea LM, Beck EJ, Probst YC et al. (2017) Whole grain intake of Australians estimated from a cross-sectional analysis of dietary intake data from the 2011-13 Australian Health Survey. Public Health Nutr 20, 2166-2172.

10. Mann KD, Pearce MS, McKevith B et al. (2015) Low whole grain intake in the UK: results from the National Diet and Nutrition Survey rolling programme 2008-11. Br J Nutr 113, 1643-1651.

11. Bellisle F, Hebel P, Colin J et al. (2014) Consumption of whole grains in French children, adolescents and adults. BrJ Nutr 112, 1674-1684.

12. O'Donovan CB, Devlin NF, Buffini M et al. (2019) Whole grain intakes in Irish adults: findings from the National Adults Nutrition Survey (NANS). Eur J Nutr 58, 541-550.

13. Kyro C, Skeie G, Dragsted LO et al. (2012) Intake of whole grain in Scandinavia: intake, sources and compliance with new national recommendations. Scand Public Health 40, 76-84.

14. Sette S, D'Addezio L, Piccinelli R et al. (2017) Intakes of whole grain in an Italian sample of children, adolescents and adults. Eur J Nutr 56, 521-533.

15. Jacobs DR Jr, Andersen LF \& Blomhoff R (2007) Whole grain consumption is associated with a reduced risk of noncardiovascular, non-cancer death attributed to inflammatory diseases in the Iowa Women's Health Study. Am J Clin Nutr 85, 1606-1614.

16. Jacobs DR Jr, Meyer KA, Kushi LH et al. (1999) Is whole grain intake associated with reduced total and cause-specific death 
rates in older women? The Iowa Women's Health Study. Am J Public Health 89, 322-329.

17. Steffen LM, Jacobs Jr DR, Stevens J et al. (2003) Associations of whole-grain, refined-grain, and fruit and vegetable consumption with risks of all-cause mortality and incident coronary artery disease and ischemic stroke: the Atherosclerosis Risk in Communities (ARIC) Study. Am J Clin Nutr 78, 383-390.

18. Jacobs DR Jr, Meyer HE \& Solvoll K (2001) Reduced mortality among whole grain bread eaters in men and women in the Norwegian County Study. Eur J Clin Nutr 55, 137-143.

19. Sahyoun NR, Jacques PF, Zhang XL et al. (2006) Wholegrain intake is inversely associated with the metabolic syndrome and mortality in older adults. Am J Clin Nutr 83, 124-131.

20. He M, van Dam RM, Rimm E et al. (2010) Whole-grain, cereal fiber, bran, and germ intake and the risks of all-cause and cardiovascular disease-specific mortality among women with type 2 diabetes mellitus. Circulation 121, 2162-2168.

21. Ferruzzi MG, Jonnalagadda SS, Liu S et al. (2014) Developing a standard definition of whole-grain foods for dietary recommendations: summary report of a multidisciplinary expert rountable discussion. Adv Nutr 5, 164-176.

22. Wu H, Flint AJ, Qi Q et al. (2015) Whole grain intake and mortality: two large prospective studies in U.S. Men and women. JAMA Intern Med 175, 373-384.

23. U.S. Food and Drug Administration (FDA) (1999) Health claim notification for whole grain foods. https://www.fda.gov/ Food/LabelingNutrition/ucm073639.htm (accessed Feburary 2019)

24. American Association of Cereal Chemists International (AACCI) (2013) AACCI's Whole Grains Working Group unveils new whole grain products characterization. http:// www.aaccnet.org/initiatives/definitions/pages/wholegrain. aspx (accessed Feburary 2019).

25. U.S. Department of Agriculture \& U.S. Department of Health and Human Services (2010) Dietary Guidelines for Americans, 7 ed. Washington, DC: U.S. Government Printing Office.

26. Kranz S, Dodd KW, Juan WY et al. (2017) Whole grains contribute only a small proportion of dietary fiber to the U.S. diet. Nutrients 9, 153.

27. Jacobs Jr DR, Meyer KA, Kushi LH et al. (1998) Whole-grain intake may reduce the risk of ischemic heart disease death in postmenopausal women: the Iowa Women's Health study. Am J Clin Nutr 68, 248-257.

28. Ross AB, van der Kamp J-W, King R et al. (2017) Perspective: a definition for whole-grain food products - Recommendations from the Healthgrain Forum. Adv Nutrition 8, 525-531.

29. Australian Bureau of Statistics (ABS) (2013) Australian Health Survey: User's Guide 2011-13 cat. no. 4363.0.55.001. Canberra: ABS. https://www.abs.gov.au/ ausstats/abs@.nsf/mf/4363.0.55.001 (accessed April 2018).

30. Australian Bureau of Statistics (ABS) (2014) Australian Health Survey: Nutrition First Results - Foods and Nutrients 2011-12 cat. no. 4364.0.55.007. Canberra: ABS. https://www.abs.gov.au/ausstats/abs@.nsf/lookup/4364.0.55. 007main+features12011-12 (accessed July 2018).

31. Food Standards Australia New Zealand (FSANZ) (2014) AUSNUT 2011-13 Australian Food Composition Database. https://www.foodstandards.gov.au/science/monitoring nutrients/ausnut/Documents/8b.\%20AUSNUT\%202011-13 \%20AHS\%20Food\%20Nutrient\%20Database.xls (accessed April 2018)

32. Dalton SMC, Probst YC, Batterham MJ et al. (2014) Compilation of an Australian database of manufactured and packaged food products containing wholegrain ingredients. J Food Comp Anal 36, 24-34.
33. Galea LM, Dalton SMC, Beck EJ et al. (2016) Update of a database for estimation of whole grain content of foods in Australia. J Food Comp Anal 50, 23-29.

34. Australian Bureau of Statistics (ABS) (2014) Australian Health Survey: Users Guide 2011-13 - Discretionary Food List. https://www.abs.gov.au/AUSSTATS/subscriber.nsf/log?open agent\&Discretionary\%20food\%20list.xls\&4363.0.55.001\&Data \%20Cubes\&C38E7117796E8066CA257CD200147FE1\&0\& 2011-13\&13.05.2014\&Latest (accessed April 2018).

35. Australian Bureau of Statistics (ABS) (2014) Australian Health Survey: Users Guide, 2011-13: Discretionary Foods cat. no. 4363.0.55.001. Canberra: ABS. https://www.abs. gov.au/ausstats/abs@.nsf/Lookup/BA1526F0D19FA21DCA 257CD2001CA166?opendocument (accessed July 2018).

36. National Health and Medical Research Council (NHMRC) (2017) Nutrient Reference Values Summary Tables. Canberra: National Health and Medical Research Council (NHMRC). https://www.nrv.gov.au/sites/default/files/content/resources/ 1.21\%20Summary\%20Tables\%20-\%20updated\%20August $\% 20$ 2017.xls (accessed May 2018).

37. Haubrock J, Nothlings U, Volatier J-L et al. (2011) Estimating usual food intake distributions by using the multiple source method in the EPIC-Potsdam Calibration Study. J Nutr 141, 914-920.

38. EFCOVAL Consortium (2011) Multiple Source Method (MSM) for estimating usual dietary intake from short term measurement data: User Guide. https://msm.dife.de/static/MSM_ UserGuide.pdf (accessed April 2018).

39. Barrett EM, Probst YC \& Beck EJ (2018) Cereal fibre intake in Australia: a cross-sectional analysis of the 2011-12 National Nutrition and Physical Activity Survey. Int J Food Sci Nutr 69, 619-627.

40. Food Standards Australia New Zealand (FSANZ) (2014) AUSNUT 2011-13 Food and Dietary Supplement Classification System. https://www.foodstandards.gov.au/science/monitoring nutrients/ausnut/Documents/8i.\%20Australian\%20Health\%20 Survey\%20Classification\%20System.xls (accessed April 2018).

41. Australian Government (1905) Census and Statistics Act. Australia. https://www.legislation.gov.au/Details/C2016C0 1005 (accessed October 2018).

42. Thane CW, Jones AR, Stephan AM et al. (2007) Comparative whole-grain intake of British adults in 1986-7 and 2000-1. Br J Nutr 97, 987-992.

43. Australian Bureau of Statistics (ABS) (2014) Australian Health Survey: Nutrition First Results - Foods and Nutrients, 2011-12: Discretionary Foods cat. no. 4364.0.55.007. Canberra: ABS. https://www.abs.gov.au/ausstats/abs@.nsf/ Lookup/by\%20Subject/4364.0.55.007 2011-12 Main\%20 Features Discretionary\%20foods 700 (accessed July 2018).

44. Story M, Neumark-sztainer D \& French S (2002) Individual and environmental influences on adolescent eating behaviours. J Am Diet Assoc 102, Suppl. 3, S40-S51.

45. Liese AD, Roach AK, Sparks KC et al. (2003) Whole grain intake and insulin sensitivity: the insulin resistance atherosclerosis study. Am J Clin Nutr 78, 965-971.

46. Bazzano LA, Song Y, Bubes V et al. (2005) Dietary intake of whole and refined grain breakfast cereals and weight gain in men. Obes Res 13, 1952-1960.

47. Li B, Zhang G, Tan M et al. (2016) Consumption of whole grains in relation to mortality from all causes, cardiovascular disease and diabetes: dose-response metaanalysis of prospective cohort studies. Medicine (Baltimore) 95, e4229.

48. Franz M \& Sampson L (2006) Challenges in developing a whole grain database: definitions, methods and quantification. J Food Compos Anal 19, Suppl. 1, s38-s44.

49. Devlin NFC, McNulty BA, Gibney MJ et al. (2013) Whole grain intakes in the diets of Irish children and teenagers. BrJ Nutr 110, 354-362. 